Journal of

\section{Applied Crystallography}

ISSN 0021-8898

Received 2 November 2012

Accepted 4 February 2013

\title{
X-ray characterization of Ge dots epitaxially grown on nanostructured Si islands on silicon-on-insulator substrates
}

\author{
Peter Zaumseil, ${ }^{\text {a* }}$ Grzegorz Kozlowski, ${ }^{a}$ Yuji Yamamoto, ${ }^{a}$ Markus Andreas \\ Schubert $^{\mathrm{a}}$ and Thomas Schroeder ${ }^{\mathrm{a}, \mathrm{b}}$ \\ a IHP, Im Technologiepark 25, Frankfurt (Oder), 15236, Germany, and ${ }^{\mathbf{b}}$ Brandenburgische \\ Technische Universität Cottbus, Konrad-Zuse-Strasse 1, Cottbus, 03046, Germany. Correspon- \\ dence e-mail: zaumseil@ihp-microelectronics.com
}

\begin{abstract}
On the way to integrate lattice mismatched semiconductors on $\mathrm{Si}(001)$, the $\mathrm{Ge} /$ $\mathrm{Si}$ heterosystem was used as a case study for the concept of compliant substrate effects that offer the vision to be able to integrate defect-free alternative semiconductor structures on $\mathrm{Si}$. Ge nanoclusters were selectively grown by chemical vapour deposition on Si nano-islands on silicon-on-insulator (SOI) substrates. The strain states of Ge clusters and $\mathrm{Si}$ islands were measured by grazing-incidence diffraction using a laboratory-based X-ray diffraction technique. A tensile strain of up to $0.5 \%$ was detected in the Si islands after direct Ge deposition. Using a thin $(\sim 10 \mathrm{~nm}) \mathrm{SiGe}$ buffer layer between $\mathrm{Si}$ and Ge the tensile strain increases to $1.8 \%$. Transmission electron microscopy studies confirm the absence of a regular grid of misfit dislocations in such structures. This clear experimental evidence for the compliance of Si nanoislands on SOI substrates opens a new integration concept that is not only limited to Ge but also extendable to semiconductors like III-V and II-VI materials.
\end{abstract}

\section{Introduction}

The integration of alternative semiconductor materials on silicon is an important materials science approach to increase performance and/or functionality of $\mathrm{Si}$ microelectronics. InGaAs heterostructures have been investigated as highmobility n-channel materials for scaled complementary metal oxide semiconductor (CMOS) transistors (Sun et al., 2007), and Ge photonic modules (e.g. ultra-fast photodetectors) have been integrated in $\mathrm{Si}$ CMOSs to merge electronics and photonics on a single Si chip (DeRose et al., 2011).

Advanced selective heteroepitaxy techniques to enable lattice mismatched semiconductor integration on $\mathrm{Si}(001)$ are thus of fundamental importance. The Ge/Si system is probably the most extensively investigated material combination. The integration of Ge may be done either globally over the whole $\mathrm{Si}$ wafer to set up so-called virtual Ge substrates on $\mathrm{Si}$ or locally by selective growth techniques to limit Ge thin-film deposition to the area of the future device.

Owing to the large lattice mismatch between $\mathrm{Ge}$ and $\mathrm{Si}$ of about $4.2 \%$, a global deposition of $\mathrm{Ge}$ on $\mathrm{Si}$ will be accompanied unavoidably by the formation of misfit dislocations at the interface. Therefore, much effort has been made to reduce at least the number of threading dislocations that are responsible for the degradation of electrical properties of $\mathrm{Ge}$. Thick graded SiGe buffer layers were introduced (Currie et al., 1998), the direct deposition of $\mathrm{Ge}$ on $\mathrm{Si}$ was optimized by annealing steps during/after the Ge layer growth (Hartmann $e t$ al., 2005; Choi et al., 2008; Yamamoto et al., 2011), heteroepitaxially grown rare earth oxides like $\mathrm{Pr}_{2} \mathrm{O}_{3}$ were used as a buffer layer on blanket Si wafers (Giussani et al., 2009), or patterned $\mathrm{Si}(001)$ substrates with growth windows in $\mathrm{SiO}_{2}$ masks were used (Fitzgerald, 1989; Park et al., 2007).

An alternative approach to the classical thin-film deposition is selective Ge growth on nanostructured Si substrates. The basics of this so-called nano-heteroepitaxy (NHE) were developed by Zubia \& Hersee (1999) and Zubia et al. (2000). The nanostructured substrate acts as a compliant material that accommodates part of the misfit strain of the epilayer by an elastic response on the growing film. Under ideal conditions, this may result in a film growth completely free of misfit dislocations.

In our previous studies we tried to apply the NHE approach to the $\mathrm{Ge} / \mathrm{Si}$ system. A chemical-vapour-deposition-based process was used to grow Ge selectively on Si nano-pillars of different dimensions (Zaumseil et al., 2011; Kozlowski et al., 2011, 2012). Clear compliance behaviour of the Si substrate was not achieved in these experiments, mainly owing to the $\mathrm{SiO}_{2}$ growth mask, which is necessary to protect the side walls of the $\mathrm{Si}$ nanostructures and interspaces from unwanted $\mathrm{Ge}$ deposition. A weak improvement was obtained by the use of thin SiGe buffer layers (Zaumseil, Kozlowski, Schubert et al., 2012). In a second approach we investigated nanostructured $\mathrm{Si}$ islands on silicon-on-insulator (SOI) substrates by advanced third-generation synchrotron radiation techniques and found 
clear experimental evidence for the compliance of Si nanoislands (Zaumseil, Kozlowski, Yamamoto et al., 2012).

In this paper we demonstrate that the strain characterization of nanostructured Si islands on SOI substrates with twodimensional periodic arrangement is also possible by the use of a laboratory-based X-ray diffraction technique only. We show in a 'proof of principle' study that by the use of $\sim 10 \mathrm{~nm}$ thick $\mathrm{Si}_{(1-x)} \mathrm{Ge}_{x}$ buffer layers the compliance behaviour of the $\mathrm{Si}$ islands can be significantly improved up to about $1.8 \%$ tensile strain, which is nearly half the misfit value between $\mathrm{Ge}$ and $\mathrm{Si}$.

\section{Experimental details}

SOI wafers of (001) orientation and $200 \mathrm{~mm}$ diameter with $54 \mathrm{~nm}$ box $\mathrm{Si}$ and $145 \mathrm{~nm} \mathrm{SiO}{ }_{2}$ were used for the preparation of Si nano-islands with Ge dots on top. The thickness of the box Si of some wafers was reduced to $27 \mathrm{~nm}$ by wet oxidation and HF etching. Standard procedures of lithographic structuring followed by dry etching were used to generate a twodimensional pattern of $\mathrm{Si}$ islands of different diameter with $360 \mathrm{~nm}$ periodicity. The free-standing $\mathrm{SiO}_{2}$ thickness is reduced in this process by $\sim 37 \mathrm{~nm}$. The side walls of the $\mathrm{Si}$ islands were cleaned by growth of $10 \mathrm{~nm} \mathrm{SiO}_{2}$, which was removed afterwards by etching.

Fig. 1(a) shows a typical scanning electron microscopy (SEM) image of $\sim 125 \mathrm{~nm}$-diameter $\mathrm{Si}$ islands sitting on

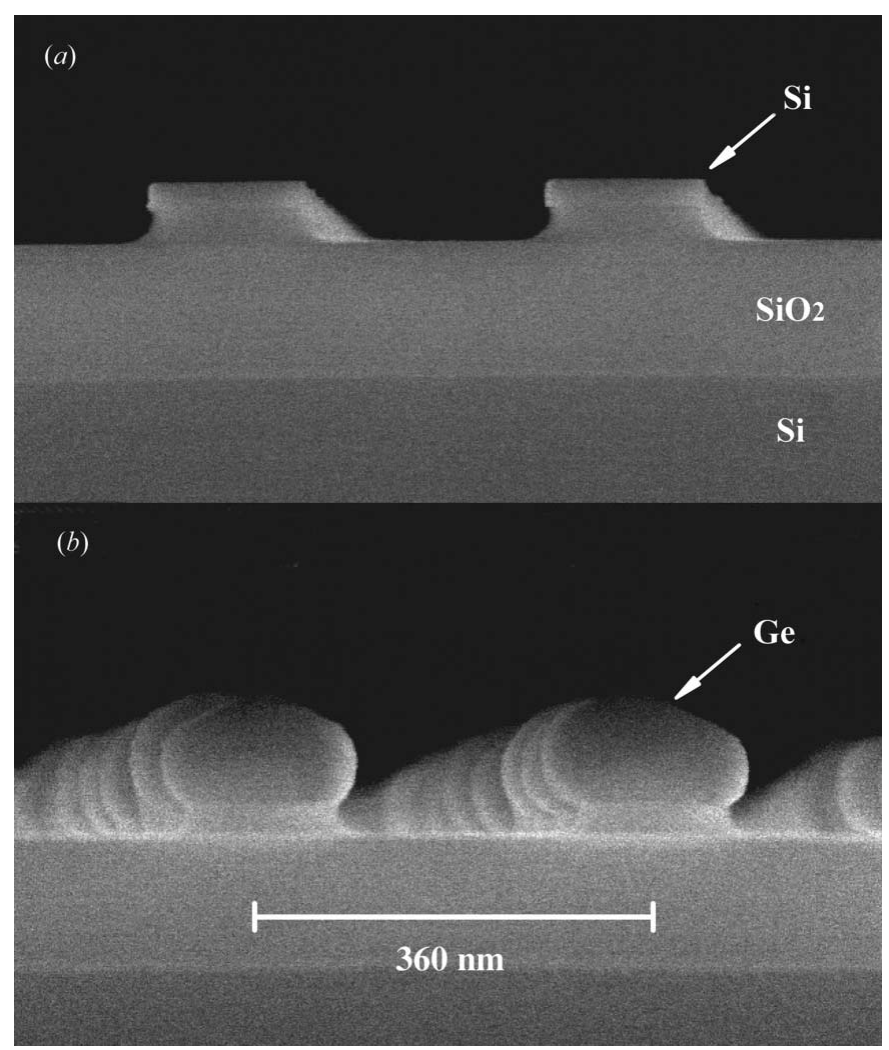

Figure 1

SEM micrographs of two samples with $27 \mathrm{~nm}$-thick Si islands of $125 \mathrm{~nm}$ diameter on $145 \mathrm{~nm} \mathrm{SiO}$ in the pre-deposited state $(a)$ and after $\mathrm{Ge}$ deposition and annealing at $1023 \mathrm{~K}(b)$.
$40 \mathrm{~nm}$-high $\mathrm{SiO}_{2}$ mesa structures after nitride removal and final cleaning. After a short prebake at $1123 \mathrm{~K}$ in a reducedpressure chemical vapour deposition reactor, Ge was deposited either directly on the $\mathrm{Si}$ islands by a two-step process, with a Ge seed layer grown at $573 \mathrm{~K}$ followed by the Ge growth process at $823 \mathrm{~K}$, or on $10 \mathrm{~nm}$-thick $\mathrm{Si}_{(1-x)} \mathrm{Ge}_{x}$ buffer layers deposited at $873 \mathrm{~K}$ with nominal Ge content $x=0.6$ and 0.3 . The calibration of the Ge deposition was done on blanket bulk $\mathrm{Si}$ wafers so that the real values for structured SOI wafers might be different. Post-deposition annealing was performed at 973,1023 or $1073 \mathrm{~K}$ for $1 \mathrm{~min}$ in an $\mathrm{H}_{2}$ atmosphere. Fig. 1(b) shows a SEM micrograph after direct Ge deposition and annealing at $1023 \mathrm{~K}$.

All of the grazing-incidence X-ray diffraction (GID) measurements were performed with a Rigaku SmartLab diffractometer equipped with a $9 \mathrm{~kW}$ rotating anode using $\mathrm{Cu} K \alpha$ radiation. This diffractometer allows a sample to be kept in a horizontal position during the measurements. An automatic alignment procedure is used to adjust the sample surface normal parallel to the $\varphi$ rotation axis of the diffractometer with an accuracy of about $0.002^{\circ}$. This guarantees a well defined and constant angle of incidence independent of the sample in-plane orientation. A Ge(400) two-crystal collimator uses the $\mathrm{Cu} K \alpha_{1}$ wavelength only and reduces the beam divergence in the direction of specular diffraction to about $0.003^{\circ}$. The divergence of the incident beam in the direction of the GID diffraction is limited by a $0.5^{\circ}$ Soller slit, while a $0.114^{\circ}$ Soller slit system in front of the detector determines mainly the angular resolution of the total arrangement. The incoming beam has a horizontal width of $5 \mathrm{~mm}$ and a height of $0.1 \mathrm{~mm}$. The diffracted beam is measured with $5 \mathrm{~mm}$ horizontal width and $3 \mathrm{~mm}$ open vertical slits (integral scheme in exit angle $\alpha_{\mathrm{f}}$ ). The obtained resolution is $0.08^{\circ}$ FWHM for a $\varphi$ rotation and $0.15^{\circ}$ for a $2 \theta / \varphi$ rotation for the 400 reflection of a perfect Si sample. For absolute measurements of in-plane lattice parameters, the angular scale of the diffractometer was always checked (calibrated) with the signal of the Si substrate.

SEM images were obtained with an S4500-II from Hitachi, and transmission electron microscopy (TEM) studies were carried out using an FEI Tecnai Osiris instrument.

\section{Results and discussion}

The strain state characterization of the Si nano-islands, which is one of the main goals of this study, requires a clear separation between the signals from $\mathrm{Si}$ islands and $\mathrm{Si}$ substrate. Two features are of advantage in this respect, as demonstrated in Fig. 2. Taking the diffracted intensity of the in-plane 400 reflection as a function of the angle of incidence $\alpha_{\mathrm{i}}$, the $\mathrm{Si}$ islands on top of the used material stack contribute from low angles on, while the $\mathrm{Si}$ substrate gives a clear signal above the critical angle of the $\mathrm{SiO}_{2}$ layer of about $0.22^{\circ}$ only. Furthermore, the crystal lattices of the Si substrate and the $\mathrm{Si}$ box layer do not have exactly the same in-plane orientation. Scans in $\varphi(\varphi$ axis is parallel to the [001] substrate lattice normal) at the same Bragg angle but $\alpha_{\mathrm{i}}$ values suited for $\mathrm{Si}$ islands and substrate, respectively, confirm a misorientation of 
some tenth of a degree, with slight differences for individual wafers. Thus it is possible to be especially sensitive to the $\mathrm{Si}$ islands by selection of proper $\varphi$ and $\alpha_{\mathrm{i}}$ values. On the other hand, the Si substrate signal can be used to calibrate the diffractometer.

The in-plane lattice parameter $a_{0}$ determined from the peak position of the $400 \mathrm{Si}$ island reflection for structures without Ge deposition is, within the error limits, very close to the bulk $\mathrm{Si}$ value for all investigated samples. Calculated strain values $\varepsilon_{0}=\left(a_{0}-a_{\mathrm{Si}}\right) / a_{\mathrm{Si}}$ vary between -0.02 and $+0.11 \%$.

Fig. 3(a) shows a typical $2 \theta / \varphi$ scan of the 400 reflection for a sample with Si islands of about $125 \mathrm{~nm}$ diameter and $54 \mathrm{~nm}$ thickness with Ge directly deposited on it in the as-deposited state. Similar curves were measured for incident angles between 0.02 and $0.32^{\circ}$. In this case, the curve can be fitted by four Gaussian profiles that are marked as $\mathrm{Ge}, \mathrm{SiGe}_{1}, \mathrm{SiGe}_{2}$ and $\mathrm{Si}$ islands.

The in-plane lattice parameters calculated from the corresponding peak position are plotted as a function of $\alpha_{\mathrm{i}}$ in Fig. 3(b). The results of different measurements are in very
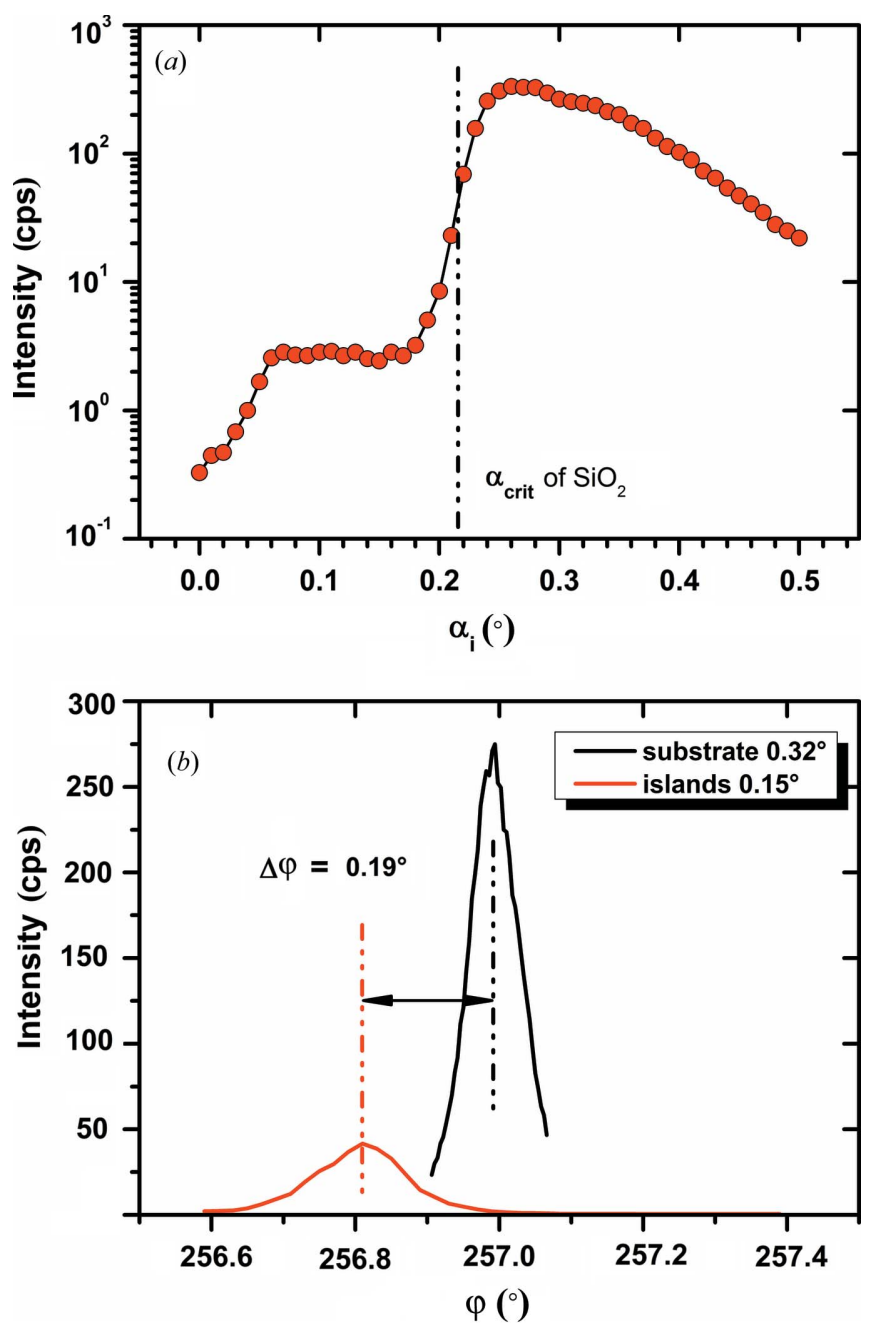

Figure 2

(a) Intensity of in-plane Si 400 diffraction versus angle of incidence $\alpha_{\mathrm{i}}$ for a sample with $\mathrm{Si}$ islands only and $(b)$ its dependence on in-plane orientation $\varphi$ for Si substrate and islands. good agreement, and the averaged values of such a set of data increase the accuracy and reliability significantly compared with a single measurement. The highest signal is related to the Ge dot. Supposing that the diffracting material is pure Ge, which was confirmed by specular 004 measurements (not shown), the slightly smaller in-plane lattice parameter compared with the Ge bulk value indicates a compressive strain of about $-0.17 \%$. The next peak $\left(\mathrm{SiGe}_{1}\right)$ is obviously the result of fitting the slightly asymmetric Ge peak within the four-curve model and can be explained by two mechanisms: (i) the in-diffusion of $\mathrm{Si}$ that forms an SiGe alloy with high $\mathrm{Ge}$ content and/or (ii) the existence of stronger compressively strained parts of the Ge dots.

The pronounced $\mathrm{SiGe}_{2}$ peak indicates the existence of a significant volume fraction in the grown $\mathrm{Ge}$ that has formed an SiGe alloy with a relative constant Ge content of about $42 \%$. Owing to possible additional strain components, this value is only a rough estimate. The fact that this peak is well separated from the $\mathrm{Ge} / \mathrm{SiGe}_{1}$ peak on one side and the $\mathrm{Si}$ islands peak on the other side shows that there does not exist a smooth tran-
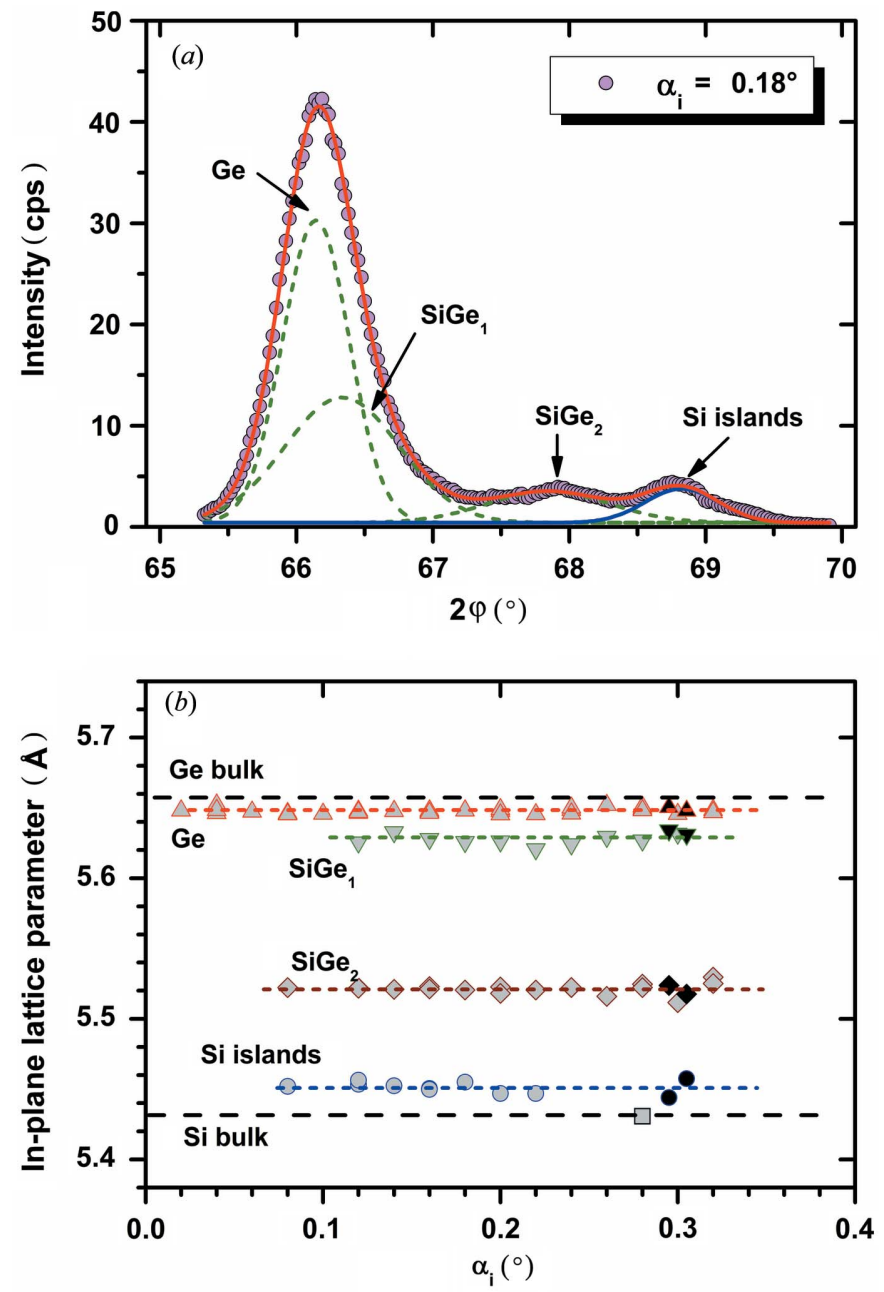

Figure 3

(a) In-plane 400 diffraction curve at $\alpha_{\mathrm{i}}=0.18^{\circ}$ and its fitting by four Gaussian profiles and $(b)$ the estimated in-plane lattice parameters for similar fittings at different $\alpha_{\mathrm{i}}$ values. The data shown with black filled symbols were obtained by synchrotron measurements of 400 and 620 diffraction for comparison. 


\section{X-ray diffraction and imaging}

sition of Si content from the $\mathrm{Si}$ islands to the pure Ge as would be expected for a thermal diffusion process. This fragment of SiGe volume might be the result of a strain-driven interdiffusion process in the early stages of Ge growth.

The in-plane lattice constant calculated from the Si islands peaks is $5.452 \AA$, which correlates to a tensile strain of the $\mathrm{Si}$ islands of $+0.39 \%$. It was demonstrated by synchrotron energy dispersive X-ray diffraction studies (Zaumseil, Kozlowski, Yamamoto et al., 2012) that the Si islands of such structures remain pure $\mathrm{Si}$ and no detectable Ge in-diffusion takes place. Thus, this is a clear proof that nanostructured $\mathrm{Si}$ islands on SOI substrates show a compliance effect after direct deposition of Ge. Fig. 4 demonstrates in a two-dimensional plot of 400 diffraction intensity versus $2 \theta$ and $\alpha_{\mathrm{i}}$ that the signals of $\mathrm{Si}$ islands and Si substrate can be well separated for this sample. This kind of plot allows an optimal selection of $\alpha_{\mathrm{i}}$ values for

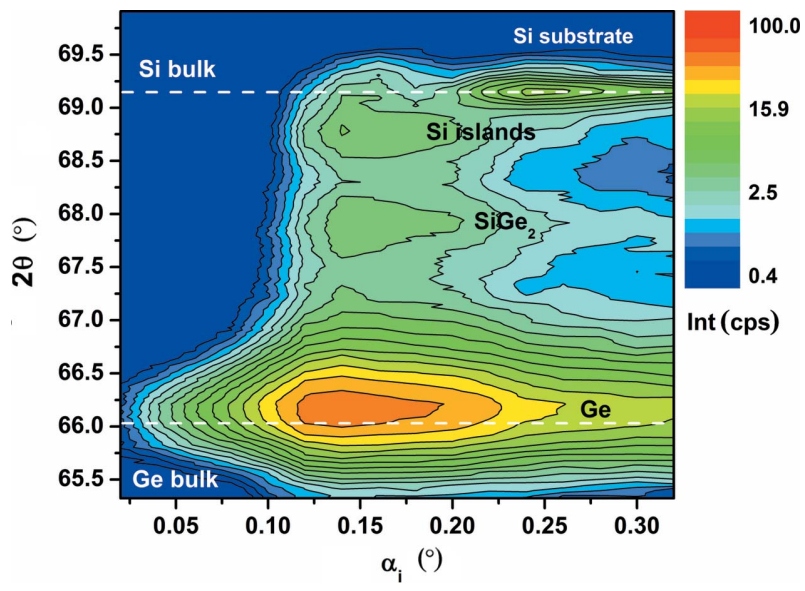

Figure 4

Intensity of in-plane 400 diffraction versus $2 \theta$ and $\alpha_{\mathrm{i}}$ of the same sample as in Fig. 3.

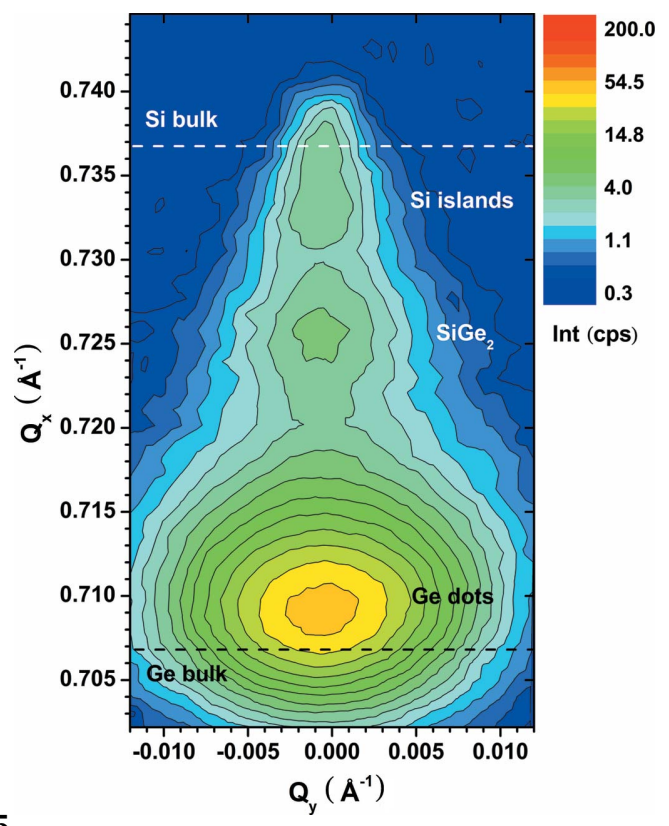

Figure 5

Reciprocal space map of 400 diffraction of the same sample as in Figs. 3 and 4 measured at $\alpha_{\mathrm{i}}=0.18^{\circ}$. the measurement of a reciprocal space map (RSM) as shown in Fig. 5.

In addition to the laboratory-based measurements, the results of 400 and 620 measurements performed at beamline ID1 at ESRF (Zaumseil, Kozlowski, Yamamoto et al., 2012) are shown in Fig. 3(b) for comparison, which demonstrates the very good agreement between both measuring techniques. A similar good agreement is shown in a comparison of the RSM obtained with a laboratory-based technique (Fig. 5) with an RSM measured at ID1 [see Fig. 2 of Zaumseil, Kozlowski, Yamamoto et al. (2012)].

Comparable investigations of samples with different $\mathrm{Si}$ island geometries show that the tensile strain can be varied to some extent. A sample with similar Ge deposition conditions and island diameter to those of the sample used for Fig. 3, but with $27 \mathrm{~nm}$ island thickness only, shows a tensile strain of $+0.52 \%$. Additional annealing leads to the tendency of decreasing strain.
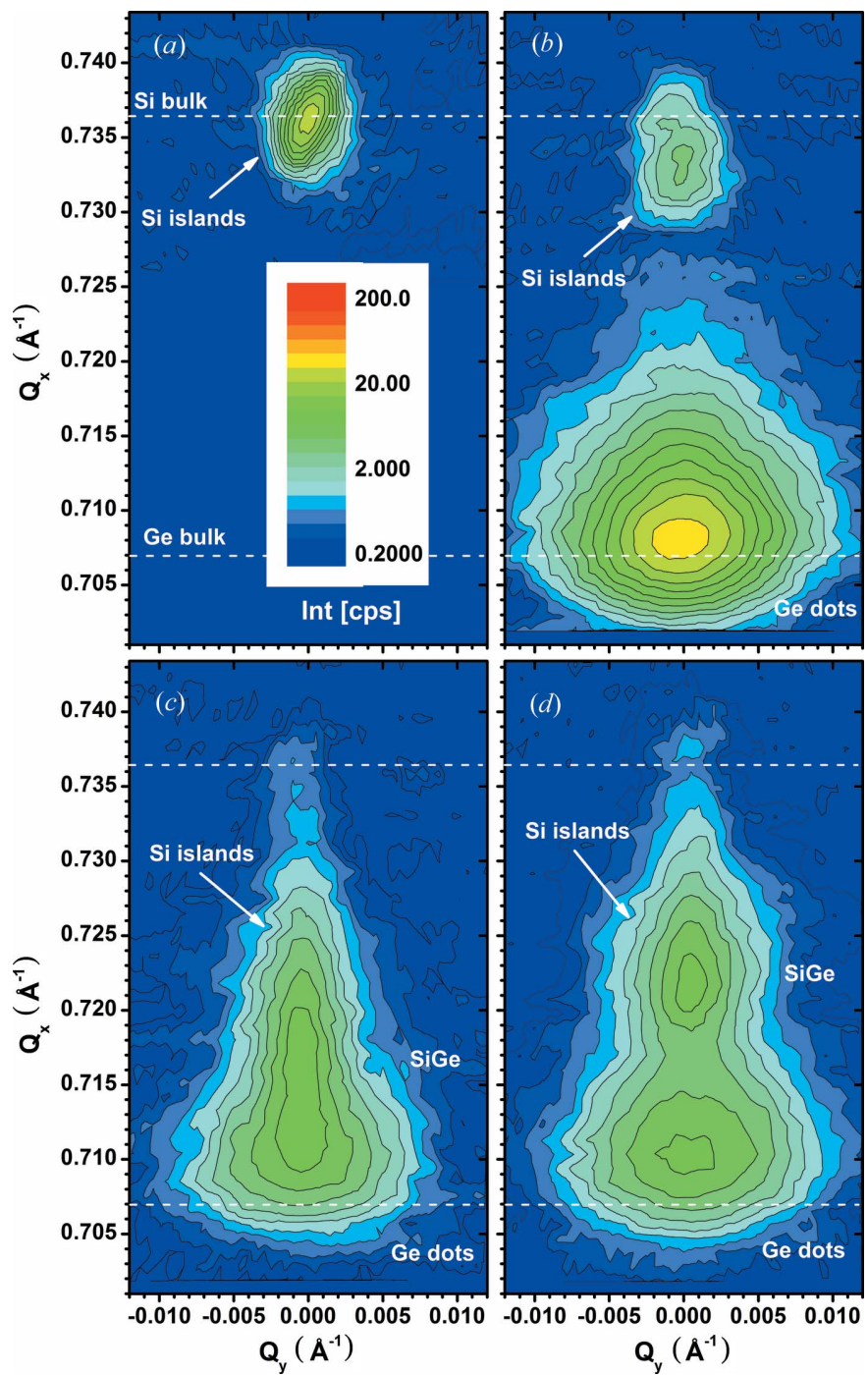

Figure 6

Comparison of reciprocal space maps of samples: $(a)$ Si islands only, $(b)$ after direct $\mathrm{Ge}$ deposition, (c) after $\mathrm{Ge}$ deposition on an $\mathrm{Si}_{0.4} \mathrm{Ge}_{0.6}$ buffer layer and $(d)$ after $\mathrm{Ge}$ deposition on an $\mathrm{Si}_{0.7} \mathrm{Ge}_{0.3}$ buffer layer in the asdeposited state measured at $\alpha_{\mathrm{i}}=0.12^{\circ}$. 
The tensile strain observed for samples with Ge directly deposited on Si nano-islands is relatively small compared with the $4.2 \%$ misfit between Ge and Si and suggests that there are still misfit dislocations generated at the $\mathrm{Ge} / \mathrm{Si}$ interface to realize the nearly full relaxation of the Ge lattice. This can be understood by the growth dynamics of this system, where a non-compliant phase must always be passed through before the compliance effect works. For Ge growth on Si the critical thickness to form misfit dislocations is only a few nanometres, and plastic relaxation occurs before sufficient strain energy is built up to strain the $\mathrm{Si}$ island.

The balance between the critical thickness to form misfit dislocations and the strain partitioning in nanostructures can be tailored for the $\mathrm{Ge} / \mathrm{Si}$ system by the use of $\mathrm{Si}_{(1-x)} \mathrm{Ge}_{x}$ with suitable Ge content $x$ (Zubia et al., 2000). The positive effect of SiGe buffer layers concerning a compliance effect on nanostructured bulk Si substrates has already been described for Si nano-pillars (Zaumseil, Kozlowski, Schubert et al., 2012).

Fig. 6 shows a set of RSMs with $27 \mathrm{~nm}$-thick Si islands (a), after direct Ge deposition (b), and after Ge deposition on
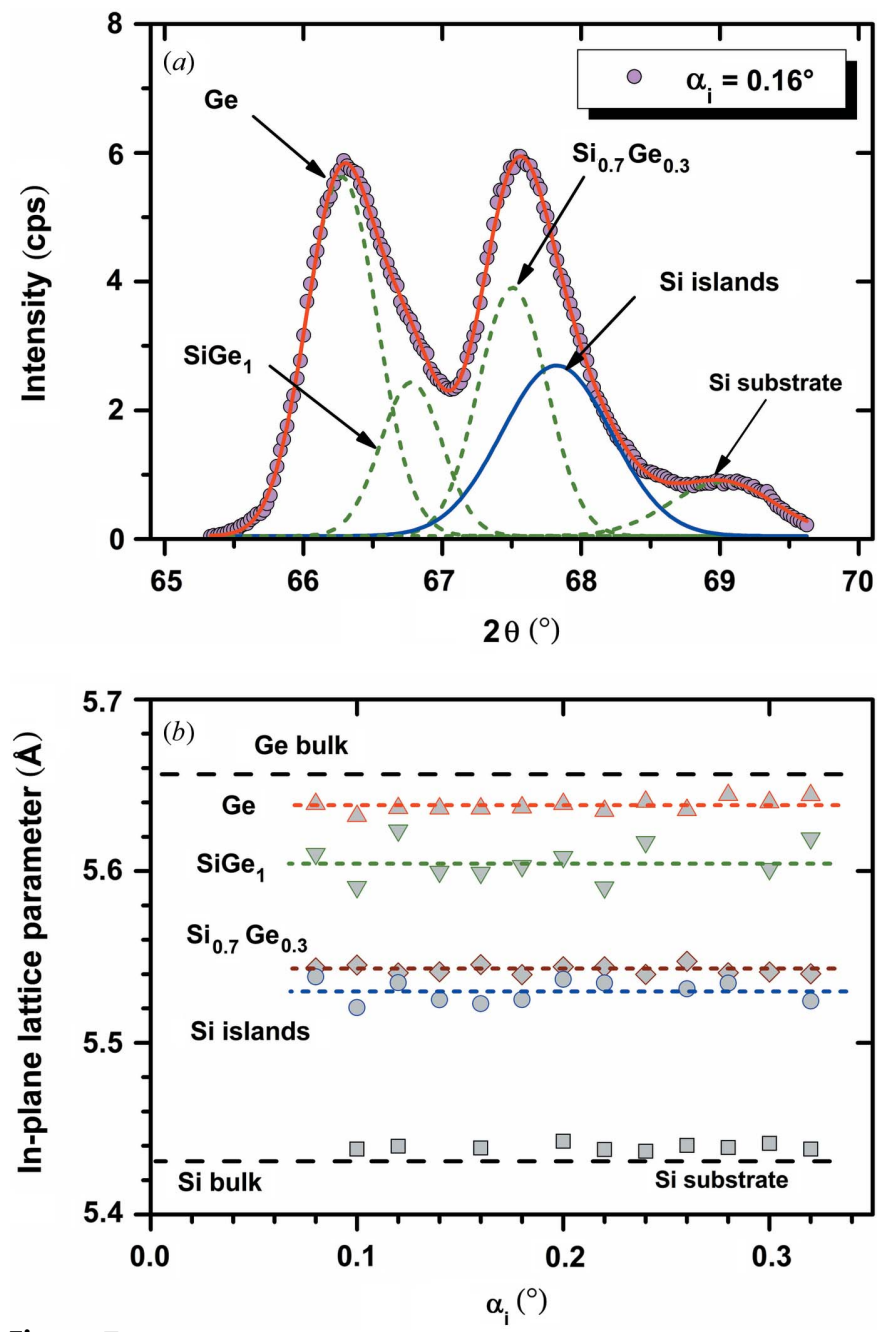

Figure 7

In-plane 400 diffraction curve at $\alpha_{\mathrm{i}}=0.16^{\circ}$ of a sample with an $\mathrm{Si}_{0.7} \mathrm{Ge}_{0.3}$ buffer layer and its fitting by five Gaussian profiles $(a)$; estimated in-plane lattice parameters for similar fittings at different $\alpha_{\mathrm{i}}$ values $(b)$.
$\mathrm{Si}_{0.4} \mathrm{Ge}_{0.6}(c)$ and $\mathrm{Si}_{0.7} \mathrm{Ge}_{0.3}(d)$ buffer layers in the as-deposited state. The position of the $\mathrm{Si}$ islands in the uncovered state agrees well with the expected position for bulk Si. After direct Ge deposition it shifts slightly towards the Ge spot to smaller $Q_{x}$ values, indicating a tensile in-plane strain. The RSM of each sample with an SiGe buffer is more complex. A separated $\mathrm{Si}$ island signal is no longer visible. Most probably it has shifted further to smaller $Q_{x}$ values and been superimposed by the SiGe buffer signal.

To detect the Si island signal, $2 \theta / \varphi$ scans of the 400 reflection were measured at different $\alpha_{\mathrm{i}}$ values and analysed by curve fitting. Fig. 7(a) shows one example for the sample with an $\mathrm{Si}_{0.7} \mathrm{Ge}_{0.3}$ buffer layer and Fig. $7(b)$ a summary of the obtained in-plane lattice parameters. Close to the peak of the $\mathrm{Si}_{0.7} \mathrm{Ge}_{0.3}$ buffer layer, there is another peak, which can be attributed to the $\mathrm{Si}$ islands. The misalignment between $\mathrm{Si}$ box and $\mathrm{Si}$ substrate lattice was relatively low for this sample so that a weak signal of the Si substrate is additionally detected. The averaged in-plane lattice parameter of the $\mathrm{Si}$ island signal is $a_{0}=5.530 \AA$, which correlates to a tensile strain of $\varepsilon_{0}=1.8 \%$. The corresponding values for a sample with an $\mathrm{Si}_{0.4} \mathrm{Ge}_{0.6}$ buffer layer are $a_{0}=5.513 \AA$ and $\varepsilon_{0}=1.5 \%$, respectively. In combination with specular $004 \mathrm{X}$-ray diffraction measurements that deliver the off-plane lattice parameter, the real $\mathrm{Ge}$ contents for the SiGe buffer layers of the two samples were determined as $x=0.51$ (instead of 0.30 ) and $x=0.70$ (instead of 0.60$)$, respectively. This demonstrates that nanostructured $\mathrm{Si}$ islands on SOI substrates require modified recipes compared with bulk Si substrates. Both buffer layers are slightly tensile strained with $\varepsilon_{0}=0.05$ and $\varepsilon_{0}=0.13 \%$, respectively. The Ge shows a slightly stronger compressive strain $(-0.34 \%)$ than in the case of direct deposition without SiGe buffer.

This shows that a significantly stronger compliance effect can be obtained by the use of thin SiGe buffer layers. These results are quite reasonable. The lower misfit between Si and SiGe results in a higher critical thickness for misfit dislocation

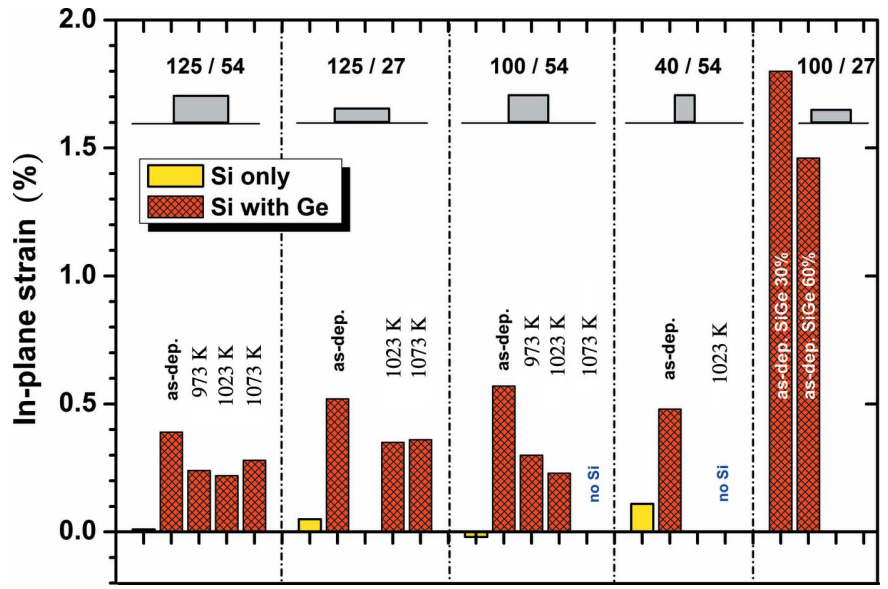

Figure 8

Summary of in-plane strain in Si islands after island preparation only and following $\mathrm{Ge}$ deposition for different island geometries and postannealing. 


\section{X-ray diffraction and imaging}

generation, which allows a stronger strain interaction with the $\mathrm{Si}$ islands. For the buffer layer with about $50 \% \mathrm{Ge}$, the inplane lattice parameters of the Si island and SiGe buffer are nearly identical, as demonstrated in Fig. 7.

Fig. 8 gives a summary of the in-plane strain state of $\mathrm{Si}$ nano-islands for different island geometries and preparation processes. It demonstrates again the strong increase of the compliance effect by the use of thin SiGe buffer layers. The use of post-deposition annealing processes, investigated for direct Ge deposition, results in the tendency to reduce the tensile strain in the Si islands. This is probably the result of a further plastic relaxation of the Ge dot structures.

In some cases with small $\mathrm{Si}$ island dimensions and/or high annealing temperatures, the Si diffraction signal disappeared completely, which indicates a significant volume reduction by dissolving processes at least below the detection limit.

Obviously, the use of a thin SiGe buffer layer leads to a measureable compliance effect in the $\mathrm{Si}$ nano-islands. To answer the key question of the structural perfection, and whether the obtained effect is sufficient to avoid the generation of misfit dislocations in the $\mathrm{Ge} / \mathrm{SiGe} / \mathrm{Si}$ material stack, TEM studies were performed. Fig. 9 shows a TEM micrograph of a sample with an SiGe buffer layer with nominal $30 \% \mathrm{Ge}$. Some of the dots with diameter $\sim 100 \mathrm{~nm}$ seem to be free of defects. At least, there is no grid of misfit dislocations visible as was the case for Ge deposited on Si nano-pillars of only $40 \mathrm{~nm}$ diameter without an SiGe buffer layer (Kozlowski et al., 2011). Other dots show structural defects, which are probably stacking faults or micro twins generated mainly at the edge of the $\mathrm{Si}$ islands that are overgrown by the Ge (Zaumseil, Kozlowski, Schubert et al., 2012; Zaumseil, Kozlowski, Yamamoto et al., 2012).

\section{Summary and conclusions}

We have demonstrated that the strain characterization of nanostructured $\mathrm{Si}$ islands on SOI substrates with two-dimensional periodic arrangement is possible by the use of a laboratory-based X-ray diffraction technique. It was con-

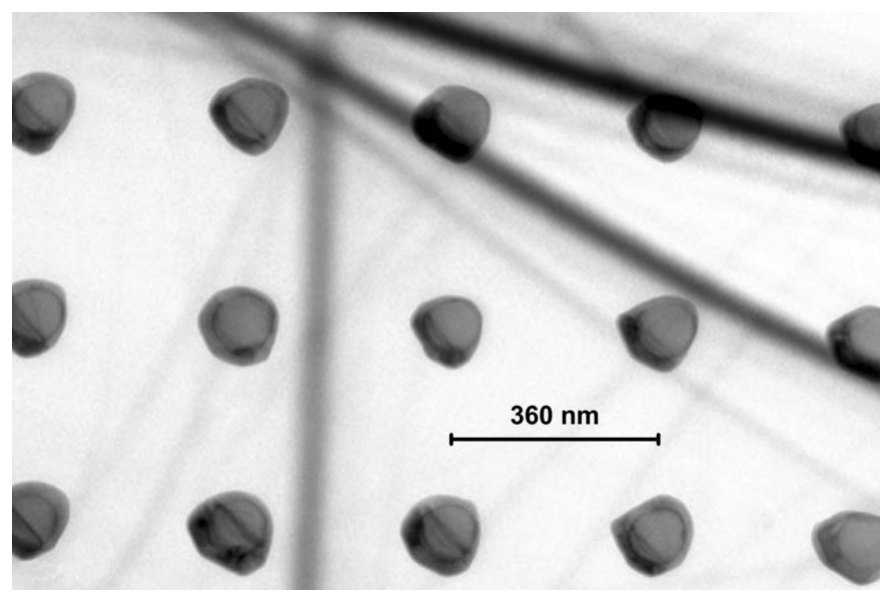

Figure 9

Plane view TEM micrograph of a sample with an $\mathrm{Si}_{0.7} \mathrm{Ge}_{0.3}$ buffer layer showing the $\mathrm{Ge} / \mathrm{SiGe} / \mathrm{Si}$ nano-islands only. firmed that the use of about $10 \mathrm{~nm}$-thick $\mathrm{Si}_{(1-x)} \mathrm{Ge}_{x}$ buffer layers improves the compliance behaviour of the $\mathrm{Si}$ islands significantly, up to $\sim 1.8 \%$ tensile strain in one example, and that it seems to be possible to grow Ge on Si free of misfit dislocations in nano-scaled structures. This 'proof of principle' study is the basis for further systematic studies to find optimal parameters concerning $\mathrm{Si}$ island geometry (diameter, thickness, eventually side wall protection), SiGe buffer parameters (Ge content, thickness) and Ge deposition (growth and annealing temperature, deposition rate etc.).

The demonstrated compliant behaviour of Si nano-islands offers a principle way toward $\mathrm{Ge} / \mathrm{Si}$ nanostructures that are free of misfit dislocations and other structural defects. Therefore it is necessary to use the structural perfection of the fabricated structures as the criterion for the above-mentioned optimization of deposition parameters. X-ray techniques may help in this process, but it requires the complex use of additional techniques like TEM and finally optical and/or electrical methods.

The better understanding of the compliance in $\mathrm{Ge} / \mathrm{Si}$ nanoheterostructures, as demonstrated in these studies, can furthermore pave the way to integrate not only high-quality Ge but also III-V and II-VI nanostructures on Si.

\section{References}

Choi, D., Ge, Y., Harris, J. S., Cagnon, J. \& Stemmer, S. (2008). J. Cryst. Growth, 310, 4273-4279.

Currie, M. T., Samavedam, S. B., Langdo, T. A., Leitz, C. W. \& Fitzgerald, E. A. (1998). Appl. Phys. Lett. 72, 1718.

DeRose, C. T., Trotter, D. C., Zortman, W. A., Starbuck, A. L., Fisher, M., Watts, M. R. \& Davids, P. S. (2011). Opt. Express, 19, 2489724904.

Fitzgerald, E. A. (1989). J. Vac. Sci. Technol. B, 7, 782.

Giussani, A., Zaumseil, P., Rodenbach, P., Weidner, G., Schubert, M. A., Geiger, D., Lichte, H., Storck, P., Wollschläger, J. \& Schroeder, T. (2009). Appl. Phys. 106, 073502.

Hartmann, J. M., Damlencourt, J.-F., Bogumilowicz, Y., Holliger, P., Rolland, G. \& Billon, T. (2005). J. Cryst. Growth, 274, 90-99.

Kozlowski, G., Zaumseil, P., Schubert, M. A., Yamamoto, Y., Bauer, J., Matejova, J., Schulli, T., Tillack, B. \& Schroeder, T. (2011). Appl. Phys. Lett. 99, 141901.

Kozlowski, G., Zaumseil, P., Schubert, M. A., Yamamoto, Y., Bauer, J., Schülli, T. U., Tillack, B. \& Schroeder, T. (2012). Nanotechnology, 23, 115704.

Park, J., Bai, J., Curtin, M., Adekore, B., Carroll, M. \& Lochtefeld, A. (2007). Appl. Phys. Lett. 90, 052113.

Sun, Y., Kiewra, E. W., Koester, S. J., Ruiz, N., Callegari, A., Fogel, K. E., Sadana, D. K., Fompeyrine, J., Webb, D. J. \& Locquet, J.-P. (2007). IEEE Electron Device Lett. 28, 473-475.

Yamamoto, Y., Zaumseil, P., Arguirov, T., Kittler, M. \& Tillack, B. (2011). Solid State Electron. 60, 2-6.

Zaumseil, P., Kozlowski, G., Schubert, M. A., Yamamoto, Y., Bauer, J., Schülli, T. U., Tillack, B. \& Schroeder, T. (2012). Nanotechnology, 23, 355706.

Zaumseil, P., Kozlowski, G., Yamamoto, Y., Bauer, J., Schubert, M. A., Schülli, T. U., Tillack, B. \& Schroeder, T. (2012). J. Appl. Phys. 112, 043506.

Zaumseil, P., Yamamoto, Y., Bauer, A., Schubert, M. A. \& Schroeder, T. (2011). J. Appl. Phys. 109, 023511.

Zubia, D. \& Hersee, S. D. (1999). J. Appl. Phys. 85, 6492.

Zubia, D., Zaidi, S. H., Hersee, S. D. \& Brueck, S. R. J. (2000). J. Vac. Sci. Technol. B, 18, 3514. 